\title{
Skeletal Muscle-Specific Overexpression of Heat Shock Protein 72 Improves Skeletal Muscle Insulin-Stimulated Glucose Uptake But Does Not Alter Whole Body Metabolism.
}

JPS Marshall ${ }^{1,2,3}$, E Estevez ${ }^{1,4}$, HL Kammoun ${ }^{1}$, EJ King ${ }^{1,5}$, CR Bruce ${ }^{6}$, BG Drew ${ }^{1,5}$, H Qian ${ }^{1}$,

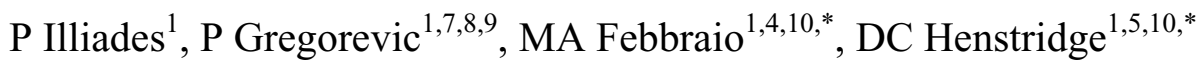

${ }^{1}$ Baker Heart and Diabetes Institute, Melbourne, Australia. ${ }^{2}$ School of Life and Environmental Science, Deakin University Melbourne, Australia. ${ }^{3}$ School of Medicine, Dentistry and Health Sciences, Melbourne University, Melbourne, Australia. ${ }^{4}$ Cellular and Molecular Metabolism Laboratory, Diabetes \& Metabolism Division, Garvan Institute of Medical Research, Sydney, Australia. ${ }^{5}$ Central Clinical School, Monash University, Melbourne, Australia. ${ }^{6}$ Institute for Physical Activity and Nutrition (IPAN), Deakin University, Geelong, Australia. ${ }^{7}$ Department of Biochemistry and Molecular Biology, Monash University, Melbourne, Australia. ${ }^{8}$ Department of Physiology, The University of Melbourne, Melbourne, Australia. ${ }^{9}$ Department of Neurology, University of Washington School of Medicine, Seattle, WA, USA. ${ }^{10}$ Address for correspondence: 1) Mark Febbraio, $\mathrm{PhD}$, Head of Division of Diabetes \& Metabolism, Garvan Institute of Medical Research, 384 Victoria St, Darlinghurst, NSW 2010, Sydney, Australia. TEL: +61 292958210 Email: m.febbraio@garvan.org.au or 2) Darren Henstridge, PhD, Baker Heart and Diabetes Institute, 75 Commercial Road, Melbourne 3004, VIC, Australia. TEL: +61 385321708 Email: darren.henstridge@baker.edu.au.*. Co last authors.

Abstract Word Count: 255

This is the author manuscript accepted for publication and has undergone full peer review but has not been through the copyediting, typesetting, pagination and proofreading process, which may lead to differences between this version and the Version of Record. Please cite this article as doi: 10.1111/dom.13319

This article is protected by copyright. All rights reserved. 
Main Body Word Count: 3873

References: 32

Tables: 0, Figures: 5 plus 4 supplementary figures

\begin{abstract}
Aims: The induction of heat shock protein 72 (Hsp72) via heating, genetic manipulation or by pharmacological activation is metabolically protective in the setting of obesity-induced insulin resistance across mammalian species. In this study, we set out to determine whether the overexpression of Hsp72, specifically in skeletal muscle, can protect against high-fat diet (HFD)-induced obesity and insulin resistance.
\end{abstract}

Materials and Methods: An Adeno-Associated Viral vector (AAV) designed to overexpress Hsp72 in skeletal muscle only, was used to study the effects of increasing Hsp72 levels on various metabolic parameters. Two studies were conducted, the first with direct intramuscular (IM) injection of the AAV:Hsp72 into the tibialis anterior hind-limb muscle and the second with a systemic injection to enable body-wide skeletal muscle transduction.

Results: IM injection of the AAV:Hsp72 significantly improved skeletal muscle insulinstimulated glucose clearance in treated hind-limb muscles compared with untreated muscles of the contralateral leg when mice were fed a HFD. Despite this finding, systemic administration of AAV:Hsp72 did not improve body composition parameters such as body weight, fat mass or percentage body fat nor did it lead to an improvement in fasting glucose levels or glucose tolerance. Furthermore, no differences were observed for other metabolic

This article is protected by copyright. All rights reserved. 
parameters such as whole-body oxygen consumption, energy expenditure or physical activity levels.

Conclusions: At the levels of Hsp72 over-expression reported herein, skeletal muscle specific Hsp72 overexpression via IM injection has the capacity to increase insulinstimulated glucose clearance in this muscle. However, upon systemic injection, which results in lower muscle Hsp72 overexpression, no beneficial effects on whole-body metabolism are observed.

\section{Introduction}

Type 2 Diabetes (T2D) is one of the most common metabolic disorders in the world and its prevalence is on the rise ${ }^{1,2}$. Predisposing factors such as obesity and physical inactivity can lead to insulin resistance, a precursor to the development of T2D. Consequently, research efforts are ongoing to identify appropriate cellular pathways to pharmacologically activate in order to combat obesity and insulin resistance and prevent the development of T2D. One of these identified targets is the inducible member of the heat shock protein 70 (HSP70) family of proteins, Hsp72, which is encoded by two genes; Hspala and Hspalb. Hsp72 is downregulated at both the gene $\mathrm{e}^{3,4}$ and protein $^{5,6}$ level in the skeletal muscle of individuals with T2D compared with healthy, non-diabetic people, while Hsp72 mRNA expression levels correlate with glucose infusion rates during a euglycemic hyperinsulinemic clamp in humans ${ }^{4}$. Furthermore, studies in humans, primates and rodents which have increased endogenous Hsp72 levels via heat treatment ${ }^{5,7-12}$, pharmacological activation ${ }^{5,13-16}$ or genetic manipulation $^{5,17}$ have all led to improvements in metabolic function while conversely, the 
loss of Hsp72 (null for Hspala and HspAlb) in mice leads to insulin resistance and mitochondrial dysfunction ${ }^{18}$.

While the precise mechanism of action as to how an elevation in Hsp72 expression leads to metabolic improvements is not fully understood, evidence suggests it could involve minimising inflammation ${ }^{5,7,10}$, altering aspects of mitochondrial function ${ }^{4,5,10,17-21}$ and/or via other yet unidentified mechanism(s). Our own work in which we overexpressed Hsp72 using Hsp72 transgenic mice (Hsp72Tg), has indicated a mitochondrial component to the mechanism of action. Increases in skeletal muscle mitochondrial oxidative enzyme activities, mitochondrial number and oxygen consumption have been observed with Hsp72 overexpression $^{5,17}$. Furthermore, we have demonstrated that Hsp72 knockout mice (Hsp72KO) have enlarged mitochondria and reduced skeletal muscle respiratory capacity ${ }^{18}$. Whilst these studies support the notion that skeletal muscle Hsp72 improves metabolism via a mitochondrial-linked process, the interpretation of the findings may have been confounded by a number of variables. Firstly, while we were aware that the Hsp72Tg model had skeletal and cardiac muscle Hsp72 overexpression without overexpression in other insulin-sensitive peripheral tissues such as the adipose tissue and the liver ${ }^{5}$, we now know that the model also has overexpression of Hsp72 in the brain. The potential exists, therefore, that the observed improvements in metabolic control originated from the brain rather than skeletal muscle. This likelihood is amplified by the finding from metabolic caging analysis that indicated the Hsp72Tg mice had increased physical activity levels which may have been driven by the brain Hsp72 overexpression. Further, it is unknown whether there is a critical level of 
expression of Hsp72 that is needed to be reached for metabolic effects to be observed. Consequently, in order to, determine whether skeletal muscle-specific induction of Hsp72 leads to protection against HFD-induced obesity and insulin resistance, independently of any central or physical activity-dependent effects, we aimed to perform a series of experiments to specifically examine the metabolic consequences of muscle-specific Hsp72 overexpression using adeno-associated viral technology.

\section{Materials and Methods}

\section{Animals}

All activities involving the use of animals for research were approved by the Alfred Medical Research Education Precinct Animal Ethics Committee (AMREP AEC) and conducted according to the guidelines of the National Health and Medical Research Council of Australia for animal experimentation. For the metabolic experiments (IM and systemic) we have included flow charts for the reporting of animal use and analysis in preclinical studies based on the template proposed by Drucker ${ }^{22}$ (see Supp Fig 3 and 4). Studies of physical activity

This article is protected by copyright. All rights reserved. 
(beam breaks) in Fig 1A, utilized male Hsp72Tg mice carrying a transgene for the rat inducible HSP72 gene regulated by $a^{2}$-actin promoter $^{23}$. These mice were on a BALB/c background and were compared to BALB/c wildtype control mice. The data was derived as part of a previous study ${ }^{17}$ but are as yet unpublished. Studies involving the administration of AAV:Hsp72 to skeletal muscle utilised male C57BL/6J mice sourced from Alfred Medical Research and Education Precinct Animal Services. Mice were fed either a normal chow diet (NC) $(14.0 \mathrm{MJ} / \mathrm{kg}, 75.2 \% \mathrm{~kJ}$ from carbohydrate, $4.8 \%$ from fat, $20 \%$ from protein; Specialty Feeds, Glen Forrest, Western Australia, Australia) or a high fat diet (HFD) (19MJ/kg, 36\% kJ from carbohydrate, $43 \%$ from fat $21 \%$ from protein; (also high in sucrose: $20 \%$ by weight of sucrose), Specialty Feeds, Glen Forrest, Western Australia, Australia) for a total of 10 weeks, starting at 7-8 weeks of age. During the experiment, mice had free access to food and water (except for in fasting periods before a glucose tolerance test), and were housed at $22 \pm 1^{\circ} \mathrm{C}$ on a $12 \mathrm{~h}$ light/dark cycle.

\section{Production of AAV vector and delivery}

A recombinant AAV vector plasmid containing a skeletal muscle specific creatine kinase promoter (pCK6 $6^{24}$, a gift of S.D Hauschka to P. Gregorevic) and the cDNA construct for mouse Hspala (Fig 1C) was designed and generated using standard cloning techniques ${ }^{25}$, and subsequently used to prepare recombinant AAV6:Hsp72 viral vectors ${ }^{26}$. Animals received either this vector (AAV:Hsp72) or a vector containing a multiple cloning site (MCS) as control (AAV:CON). The viral vectors were administered to different cohorts of mice via two different methods. Study 1: For the intramuscular (IM) study, 30 $\mu$ L of AAV:HSP72 or AAV:CON were injected into the right or left tibialis anterior (TA) muscle, respectively at a 
dose of $1 \times 10^{11}$ vector genomes (vg). Study 2: For the systemic study, the AAV's were administered via tail vein injection at two doses, $4 \times 10^{12} \mathrm{vg}$ or $8 \times 10^{12} \mathrm{vg}$ in a total volume of $150 \mu \mathrm{L}$ using a $26 \mathrm{G}$ needle and $0.3 \mathrm{~mL}$ syringe.

\section{Body Composition Analysis}

Fat mass and lean mass were measured using a 4-in-1 EchoMRI (EchoMRI ${ }^{\mathrm{TM}}$, Houston, TX, USA) and standard laboratory scales were used for body mass (Mettler Toledo, Greifensee, Switzerland).

\section{Intravenous insulin tolerance test (ivITT)}

Intravenous insulin tolerance tests (ivITT) with glucose tracer were performed as previously described $^{27}$. Briefly, mice were anesthetized with sodium pentobarbital and their jugular vein cannulated. Following basal glucose measurements, a single injection of $0.6 \mathrm{U} / \mathrm{kg}$ lean body mass with $\left[{ }^{3} \mathrm{H}\right] 2$-deoxyglucose $(10 \mu \mathrm{Ci})$ (PerkinElmer, Waltham, MA, USA) was injected via the cannula. Blood was sampled from the tail at the indicated time points for the determination of blood glucose and plasma radioactivity of $\left[{ }^{3} \mathrm{H}\right] 2-\mathrm{DG}$. At endpoint, the TA muscles were snap frozen and stored at minus $80^{\circ} \mathrm{C}$ for analysis.

\section{Oral glucose tolerance test}

Oral glucose tolerance tests (oGTT) were performed on fasted (6 h) mice. Mice received an oral gavage of $2 \mathrm{~g}$ glucose $/ \mathrm{kg}$ lean body mass ( $25 \% \mathrm{w} / \mathrm{v}$ glucose solution) and blood glucose

This article is protected by copyright. All rights reserved. 
levels were measured via a glucometer (AccuCheck, Roche Diabetes Care, NSW, Australia) at the indicated times on the blood that was collected from the tail.

\section{Metabolic caging analyses}

A Comprehensive Laboratory Animal Monitoring System (CLAMS, Columbus Instruments, Columbus, $\mathrm{OH}, \mathrm{USA}$ ) was utilized to measure various metabolic parameters. Mice were placed into individually housed chambers and oxygen consumption $\left(\mathrm{VO}_{2}\right)$, respiratory exchange ratio (RER), Energy expenditure (heat) and total movement (beam breaks) were recorded over $48 \mathrm{hrs}$. The first $24 \mathrm{hrs}$ served as an acclimatisation period and the $24-48 \mathrm{~h}$ period served as the period we analysed.

\section{Western blot analysis}

Muscle samples were lyzed and protein concentration was determined as previously described $^{28}$. Immunoblotting was performed using the following primary antibodies: Hsp72 and Hsp90 (Enzo Life Sciences, NY, USA (formerly SRESSGEN)), FLAG and t-tubulin (Sigma Aldrich, St. Louis, MO, USA), OXPHOS cocktail (Abcam, Cambridge, UK) and GAPDH, phospho-AKT Ser 473, total AKT, Phospho-GSK-3 $\pm^{2}{ }^{2}$ (Ser21/9), total GSK-3² (27C10), from (Cell Signalling, Danvers, USA). Quantification was performed using Quantity One 1-D Analysis Software (BioRad, Hercules, CA, USA).

\section{Determination of plasma and tissue radioactivity}

Blood samples $(10 \mu 1)$ collected from mice were immediately deproteinized with barium hydroxide and zinc sulfate and $\left[{ }^{3} \mathrm{H}\right] 2-\mathrm{DG}$ radioactivity determined via liquid scintillation 
counting (Insta-Gel Plus, PerkinElmer, Waltham, MA, USA). Accumulation of $\left[{ }^{3} \mathrm{H}\right] 2-\mathrm{DG}$ radioactivity in the TA samples was determined by scintillation counting in an aqueous extract of the tissue after homogenisation. Free and phosphorylated $\left[{ }^{3} \mathrm{H}\right] 2-\mathrm{DG}$ were separated by ion exchange chromatography on Dowex 1-X8 columns (Sigma-Aldrich, St Louis, MO, USA). The area under the tracer disappearance curve for $\left[{ }^{3} \mathrm{H}\right] 2-\mathrm{DG}$ and the radioactivity for the phosphorylated $\left[{ }^{3} \mathrm{H}\right] 2-\mathrm{DG}$ from the TA muscles were used to calculate glucose concentration dependent $\left(\mathrm{Rg}\right.$ gluense concentration independent $\left(\mathrm{Kg}^{\prime}\right)$ indexes of muscle glucose uptake as previously described in mice ${ }^{29}$.

\section{Statistical Analysis}

Data are presented as mean \pm standard error of the mean (SEM). For the IM study, differences between the normal chow (NC) and high fat diet (HFD) (diet effect) were analysed via an unpaired Student's t-test. The glucose clearance data were analysed via a paired t-test as it was a within subject design. For the systemic study, two-way analysis of variance (ANOVA) was used to detect main effects for diet (NC vs. HFD) and treatment (AAV-Hsp72 vs. AAV:CON). Post-hoc analyses (Tukey's) were performed when a significant result occurred. Analyses were performed using the statistical program, Sigmastat 3.5. $\mathrm{p}<0.05$ was considered statistically significant. Asterisk indicates a diet effect and hash represents a treatment or genotype effect.

This article is protected by copyright. All rights reserved. 


\section{Results}

\section{Hsp $72 T$ mice have increased physical activity and Hsp72 brain protein expression.}

We had previously identified that Hsp72Tg mice exhibited increased whole-body $\mathrm{VO}_{2}$ and energy expenditure ${ }^{17}$, and this was a potential mechanism for the observed protection from HFD-induced weight gain and insulin resistance. A potentially contributing factor to this phenotype is an increase in physical activity levels observed in Hsp72Tg mice (Fig 1A). As the main initiator of physical activity is "central-drive", we probed brain homogenates of these Hsp72Tg mice to determine whether the transgene also promoted Hsp72 overexpression in the brain. These analyses confirmed that the Hsp72Tg model also has brain Hsp72 overexpression (Fig 1B), prompting two intriguing new potential mechanisms via which Hsp72 overexpression may be improving metabolic control in these mice. That 1) skeletal muscle Hsp72 overexpression may induce hyperactivity and 2) brain derived Hsp72 overexpression may be driving the metabolic benefits. Thus, we designed an experiment to determine the consequence of Hsp72 overexpression specifically in skeletal muscle, which would allow for the discrimination of the action of Hsp72 overexpression between the brain and skeletal muscle.

Intramuscular injection of AAV:Hsp72 partially restores $\mathrm{HFD}$-induced defective glucose clearance in skeletal muscle.

This article is protected by copyright. All rights reserved. 
We specifically designed the AAV:Hsp72 using a skeletal muscle-specific promoter (CK6) as opposed to the ubiquitous cytomegalovirus (CMV) promoter or the muscle creatine kinase (MCK) promoter, which targets all striated muscle including cardiac muscle ${ }^{24}$. Therefore, by using this construct, any findings would be restricted to the role of Hsp72 in skeletal muscle. Our first study was designed to examine whether elevated Hsp72 could improve skeletal muscle insulin-stimulated glucose clearance independently of the alterations to physical activity levels observed in the Hsp72Tg mice. Accordingly, we directly injected one tibialis anterior hindlimb muscle with the AAV:Hsp72 and the contralateral tibialis anterior muscle with AAV:CON. As mice are quadrupeds and don't hop, each leg in effect would be exposed to the same amount of physical activity, ruling this out as a potential variable. This study design is also advantageous as it allows for comparison of skeletal muscle Hsp72 overexpression in the presence of a control muscle that has the same genetics, hormonal milieu, blood flow and receives the same quantity of any treatment (i.e. in this case anaesthesia agent and insulin). Injection of the AAV:Hsp72 successfully resulted in elevated expression of Hsp72 compared to AAV:CON injection (Fig 1D, E). This was validated by blotting for the FLAG tag, which was engineered as a fusion epitope into the AAV:Hsp72 construct (Fig 1D).

To determine whether this overexpression of Hsp72 could influence glucose metabolism in a setting of obesity, insulin resistance and glucose intolerance, we fed mice either a NC or HFD for 10 weeks. As expected, body weight, fat mass and body fat percentage were increased in HFD compared with NC fed mice, while lean mass remained unchanged (Fig 2A-D). We next performed an ivITT in the presence of a radiolabelled glucose tracer $\left(\left[{ }^{3} \mathrm{H}\right] 2-\mathrm{DG}\right)$ in order 
to assess whole-body insulin tolerance and insulin-stimulated glucose clearance in skeletal muscle. While blood glucose and plasma 2-DG concentration decreased over the 30 min postinsulin stimulation in both NC and HFD cohorts, the rate and magnitude of the reduction was greater in the $\mathrm{NC}$ mice, indicating a greater insulin sensitivity in the NC fed mice (Fig 2E, F). When examining estimates of skeletal muscle glucose uptake, muscles from the HFD-fed mice exhibited reduced glucose clearance compared with $\mathrm{NC}$ fed mice (Fig 2G, H). In NC fed mice, Hsp72 overexpression did not alter rates of glucose clearance compared to AAV:CON, however in HFD, where glucose clearance was compromised, Hsp72 overexpression led to a partial restoration of glucose clearance in the AAV:Hsp72 treated muscle compared to AAV:CON (Fig 2G, H). To elucidate whether the change in muscle glucose clearance corresponded to changes in the insulin signalling cascade, we measured the phosphorylation of AKT and GSK-3. This western blot analysis (Supp Fig 2A-C) demonstrated no detectable difference between the groups, possibly reflective of the acute nature of the experimental design and the dynamic nature of this intracellular process. As mitochondrial dysfunction has been linked to skeletal muscle insulin resistance and alterations in Hsp72 expression levels has been associated altering mitochondrial function, we next measured markers of both of these processes. Measurement of proteins involved in OXPHOS (CI, II, IV and V) did not reveal any differences with diet or Hsp72 expression, suggesting no increase in mitochondrial content or these individual mitochondrial OXPHOS machinery components (Fig 2I, J).

Systemic injection of AAV:Hsp72 increases Hsp72 protein expression specifically in skeletal muscle. 
Given the encouraging results from the direct muscle injection of AAV:Hsp72, we next wanted to determine the effect of whole musculature overexpression of Hsp72 using the AAV:Hsp72. Ten weeks post systemic tail vein injection of AAV:Hsp72 at concentrations of $4 \times 10^{12}$ and $8 \times 10^{12} \mathrm{vg}$, we observed significant overexpression of Hsp72 and FLAG in gastrocnemius, quadriceps and tibialis anterior muscles when compared with AAV:CON (Fig 3A-C). Interestingly, we observed no overexpression in soleus muscle (Fig 3D), highlighting the unusual nature of this muscle compared with other muscles in mice. No overexpression was observed in other metabolic tissues including the liver, heart, white adipose tissue and brain (Fig 3E-H), suggesting that the AAV:Hsp72 mediated Hsp72 overexpression was restricted to skeletal muscle. Since there was no additive effect of using the higher AAV:Hsp72 dose, we used the $4 \times 10^{12} \mathrm{vg}$ dose to test the effect of systemically overexpressing Hsp72 on metabolic outcomes.

\section{Skeletal muscle specific overexpression of Hsp72 does not protect against HFD-induced weight gain or adiposity.}

As Hsp72Tg overexpression has been shown to protect against HFD-induced weight gain ${ }^{5,17}$, various aspects of body composition were determined 10 weeks after mice initiated a $\mathrm{NC}$ or HFD and were injected with AAV:CON or AAV:Hsp72. There was an increase in body weight, fat mass and percentage body fat (Fig 4A, C, D) in the HFD mice compared with NC, however Hsp72-overexpression did not provide any protection against this accumulation of fat. In terms of lean body mass, there was no dietary effect observed but there was a significant main effect for the AAV:Hsp72 mice to have greater lean mass (Fig 4B). 
Skeletal muscle specific overexpression of Hsp72 does not protect from HFD-induced glucose intolerance.

A number of studies have shown that Hsp72 overexpression protects against obesity-induced glucose intolerance ${ }^{5,17}$, while deletion of Hsp72 in mice ${ }^{18}$ or a decreased expression in humans correlates to an increase in insulin resistance ${ }^{3,4}$. To assess this in our model, we performed oral glucose tolerance tests (oGTT's) at five and ten weeks post AAV administration. At five weeks post injection it was evident that the HFD increased fasting glucose and induced glucose intolerance compared to those animals on a NC diet, however, AAV:Hsp72 had no effect (Fig 4E, F, G). Similarly, in mice examined ten weeks after diet implementation and AAV administration, we observed HFD-induced glucose intolerance, but no effect of the AAV:Hsp72 on improving glycaemic control in either NC or HFD fed mice (Fig 4H-J).

\section{Skeletal muscle-specific overexpression of Hsp72 does not alter metabolic parameters such} as $\mathrm{VO}_{2}, \mathrm{RER}$, energy expenditure, or physical activity levels.

Since previous studies have shown Hsp72Tg mice have a reduced RER (indicative of increased fatty acid oxidation) and higher energy expenditure and $\mathrm{VO}_{2}{ }^{17}$, we used metabolic caging (CLAMS) to determine if skeletal muscle-specific Hsp72 overexpression could alter any of these metabolic parameters. When expressed relative to the whole animal $(\mathrm{mL} / \mathrm{h}$; Fig $5 \mathrm{~A})$ or to lean mass $(\mathrm{mL} / \mathrm{kg}$ lean mass $/ \mathrm{h}$; Fig $5 \mathrm{~B}), \mathrm{VO}_{2}$ throughout both the light and dark phase as well as over the entire $24 \mathrm{~h}$ measurement period was significantly higher in the HFD-fed mice. When expressed relative to body weight, no dietary effect was observed (Fig 
5C). No matter which way the $\mathrm{VO}_{2}$ was adjusted, there was no effect of Hsp72 overexpression. This is also evidenced when graphing the $\mathrm{VO}_{2}$ data against body composition characteristics such as body weight (Fig 5D), lean mass (Fig 5E) or fat mass (Fig 5F) in a scatter plot format. RER was significantly reduced in the HFD cohorts suggestive of greater fatty acid substrate preference but was not altered by Hsp72 overexpression (Fig 5G). Energy expenditure was higher due to the increase in body weight in the high fat fed mice (Fig $5 \mathrm{H})$ while physical activity was not altered by diet or Hsp72 overexpression (Fig 5I). Collectively, the CLAMS analysis revealed no differences in energy expenditure, substrate oxidation and activity levels when overexpressing Hsp72 specifically in skeletal muscle.

\section{Comparative expression between Hsp72 overexpression models.}

One potential reason for observing improvements in glucose clearance with IM administartion but no improvement in whole-body glucose metabolism in the systemically treated mice, is that the expression of Hsp72 in the systemic experiment was not at a level high enough to elicit a metabolic response. To gauge the differences in muscle Hsp72 expression levels between the IM and systemic condition and also against those from the Hsp72Tg mice, we conducted a side by side comparison. As can be seen in Supp Fig 2A and $\mathrm{B}$, the level of expression derived from the systemic administration was markedly lower than both the IM injection and transgenic mouse models. 


\section{Discussion}

Hsp72, has emerged as a potential target for therapeutic treatment of obesity-induced insulin resistance. This is based on the observations that 1) Hsp72 levels are decreased in patients with $\mathrm{T} 2 \mathrm{D}^{3-6}$ and 2) hyperthermic treatment, which leads to a robust increase in endogenous

This article is protected by copyright. All rights reserved. 
Hsp72, has been shown to be beneficial in the maintenance of metabolic homeostasis ${ }^{5,7-12,30}$. Treatments with compounds that target Hsp72 such as Geranylgeranylacetone (GGA) ${ }^{14,15}$, matrine ${ }^{13}$, or the co-inducing compound BGP-15 $5^{16,31} 5,17$ have shown efficacy in improving metabolic control. As hyperthermic and pharmacological treatment are global in nature, identifying the precise organ(s) where elevation in Hsp72 is beneficial for protection against obesity and/or insulin resistance is of interest. Studies investigating muscle overexpression of $\mathrm{Hsp} 72^{5,17}$ have suggested it is the muscle where Hsp72 likely asserts its metabolically protective effects. However, these studies may have been confounded by a number of factors including overexpression of Hsp72 in the brain as well as by increased physical activity levels. Subsequently, the current study was designed to test the hypothesis that skeletal muscle-specific overexpression of Hsp72 improves metabolic control in a model of dietinduced obesity. While we demonstrate that increasing Hsp72 does indeed have the capacity to improve insulin-stimulated skeletal muscle glucose uptake (Study 1) in HFD mice, it has no effect on adiposity or whole-body glucose homeostasis (Study 2).

Local overexpression of Hsp72 via IM injection, led to the partial restoration of insulinstimulated glucose uptake in the AAV:Hsp72 treated leg in HFD fed mice. This finding suggests that under the conditions of a HFD, the overexpression of Hsp72 is able to somewhat buffer the muscles' ability to take up glucose into the muscle fibres. Given this finding, we progressed the studies to investigate whether whole-body skeletal muscle overexpression can protect against HFD induced obesity and glucose intolerance. In contrast with the results from the IM study, we did not find any protective effects of systemic Hsp72 skeletal muscle overexpression on any parameter tested in relation to metabolic homeostasis 
(body composition, glucose tolerance, energy expenditure, physical activity) in either NC or HFD fed mice.

There are a number of possible reasons as to why we may have observed these divergent results. Firstly, we must consider the methods employed to examine glucose homeostasis. The IM study was conducted under anaesthesia with the intravenous administration of insulin. This route of insulin administration avoids the natural route of endogenous insulin secretion and can favour insulin having a greater effect on skeletal muscle. Thus, it may have been that skeletal muscle Hsp72 is only protective in the setting of IV insulin administration. Furthermore, the potential exists that Hsp72 was only able to partially protect glucose clearance in the IM study due to the extra stress provided by the anaesthesia. Another consideration is the fact that after a meal or in this case an oral glucose bolus, skeletal muscle accounts for $\sim 30 \%$ of glucose disposal (reviewed in ${ }^{32}$ ). In our IM study we saw an improvement of $\sim 29 \%$ in the glucose clearance of the muscle on a HFD. Perhaps a $29 \%$ improvement (if it was achieved in all muscles of the body) of a component that makes up $30 \%$ of whole-body disposal is not enough of an increase to see whole body effects. Finally, as the TA muscles were able to be directly injected with the AAV, the absolute levels of overexpression of Hsp72 were higher in the IM study as compared to the systemic study and were more akin to expression levels reached with transgenic mice (Supp Fig 2). Potentially this high level of expression, similar to what is observed in transgenic models, is needed for the full effect of Hsp72 in muscle. In the current study, it was unachievable in the systemic model to achieve Hsp72 expression to the same extent as the IM model. This is evidenced by 
the higher AAV:Hsp72 dose in our pilot study (Fig 3) not increasing Hsp72 expression levels to a greater degree than the lower dose.

While previous Hsp72Tg studies have demonstrated protection against the deleterious metabolic effects of obesity, our systemic administration of AAV:Hsp72 did not reproduce such results. This could be due to a number of different factors. Firstly, Hsp72Tg mice overexpress Hsp72 from conception. Therefore, it is possible that during embryogenesis and/or early life increased Hsp72 primes the body to have increased metabolism. Secondly, we now know that the transgenic model has increased levels of Hsp72 in the brain. We are unsure of the precise consequences of this (although we do know it does not alter eating behaviour $^{5,17}$ ) but we cannot rule out that this provides some sort of neural regulation that is beneficial for metabolic processes. As the CNS controls physical activity patterns and the Hsp $72 \mathrm{Tg}$ mice have increased activity it is an intriguing thought that Hsp72 in the brain may play a role in this phenotype.

Together, despite the promising results from our IM study, our research shows that skeletal muscle specific overexpression of Hsp72 at the levels reported herein, was not sufficient to protect against HFD-induced obesity and glucose intolerance. Consequently, given these findings and our identification of brain Hsp72 overexpression in the Hsp72Tg mice, it may be warranted to study the effects of brain-specific Hsp72 elevation in relation to metabolic control and physical activity. Alternatively, there may be a critical level of skeletal muscle Hsp72 expression that is required to be reached before beneficial metabolic effects are achieved. 


\section{Acknowledgements:}

We thank the staff from the Alfred Medical Research and Education Precinct Animal Services (AMREP AS) team for their assistance and care of the mice used in this study and for the support of the Operational Infrastructure Support scheme of the Victorian State Government. We thank Tim Gardner for technical assistance and Dr Michael Cater and A/Prof Paul Adlard for student mentorship. MAF and PG are National Health and Medical Research Council of Australia (NHMRC) research fellows and DCH was supported by an Australian Diabetes Society Skip Martin Fellowship.

Conflict of Interest: MAF is Chief Scientific Officer and shareholder of N-Gene Research Laboratories, Inc.

This article is protected by copyright. All rights reserved. 


\section{Figure Legends}

Fig 1. (A) Physical activity levels in wildtype (WT) and Hsp72 Tg mice on a BALB/c background. Activity was measured by ambulatory beam breaks in a metabolic caging unit over 24 hours. \# $\mathrm{p}=<0.05$ main effect for genotype, $\mathrm{n}=5-6$ per group. (B) Brain Hsp72 protein levels measured via western blotting in Hsp72Tg or WT mice. Representative image of $n=4$ per group. (C) Diagrammatic representation of the AAV:Hsp72 used in the study. D) Representative western blots of overexpression of Hsp72 and FLAG in the TA muscle in response to AAV:Hsp72 injection compared to $\mathrm{AAV}: \mathrm{CON}$ injection. Two blots per treatment per diet displayed. (E) Quantification of Hsp72 overexpression induced by the AAV:Hsp72 compared to AAV:CON, $\mathrm{n}=5$ per condition. $* \mathrm{p}=<0.05, * * \mathrm{p}=<0.01$. Two-way ANOVA used for comparisons. Data presented as mean \pm SEM.

This article is protected by copyright. All rights reserved. 
Fig 2. Characterisation of mice used for intramuscular AAV experiments. Mice were fed a normal chow (NC) or high fat diet (HFD) for 10 weeks. (A) Body weight, (B) Fat mass, (C) Lean mass, (D) Body fat percentage. Responsiveness to intravenous insulin injection (ivITT). (E) Plasma glucose levels over 30 mins following intravenous insulin injection, (F) plasma 2deoxy-glucose counts following insulin injection. Tissue specific glucose clearance into TA muscles. (G) Skeletal muscle-specific glucose clearance (Kg) and (H) Metabolic index (Rg) $* * * \mathrm{p}<0.001, * * \mathrm{p}=<0.01, * \mathrm{p}=<0.05, \mathrm{n}=5$ per condition. Graphs indicate mean $\pm \mathrm{SEM}$. (I) Western blots of oxidative phosphorylation system (OXPHOS) complexes in the TA muscle in response to $\mathrm{AAV}: \mathrm{Hsp} 72$ injection compared to $\mathrm{AAV}: \mathrm{CON}$ injection and $\mathbf{J})$ corresponding quantification of complex expression. Complex V (ATP5A), Complex IV (UQCRC2), Complex II (SDHB) and Complex I (NDUFB8) detected, note Complex III (MTCO1) could not be detected. $* \mathrm{p}=<0.05, * * \mathrm{p}=<0.01, \mathrm{n}=5$ per group (A-H), $\mathrm{n}=6-8$ (IJ). Unpaired t-test used for NC vs HFD comparisons, paired t-test for within animal analysis (CON vs Hsp72:AAV). Graphs indicate mean \pm SEM.

Fig 3. Representative western blots demonstrating overexpression of Hsp72 and FLAG in the skeletal muscle but not in other organs in response to AAV:Hsp72 injection compared to MCS CON injection at two difference doses with respective quantification. Animals were fed a normal chow (NC) diet and were tail vein injected with $4 \times 10^{12}$ or $8 \times 10^{\wedge 12} \mathrm{vg}$ of either AAV:Hsp72 or MCS-AAV (control) and tissues were harvested after 10 weeks. Overexpression of Hsp72 was observed after injection with the AAV:HSP72 in (A) gastrocnemius (B) quadriceps and (C) tibialis anterior muscles when compared to 
AAV:CON injection. Western Blotting for FLAG additionally confirmed AAV:Hsp72 over expression. No overexpression of Hsp72 was observed in (D) soleus, (E) liver, (F) heart, (G) brain or (H) white adipose tissue. There was no significant dose effect between the two concentrations used. ** $\mathrm{p}=<0.01, \mathrm{n}=3$ per condition. Two-way ANOVA used for comparisons. Graphs indicate mean \pm SEM.

Fig 4. Body composition analysis in $\mathrm{AAV}: \mathrm{CON}$ and $\mathrm{AAV}: \mathrm{Hsp} 72$ treated mice fed a $\mathrm{NC}$ or HFD for 10 weeks (A) Body weight, (B) lean mass, (C) fat mass (D) body fat percentage, $\mathrm{n}=6$-15. Whole-body glucose metabolism analysis. Fasting blood glucose levels and glucose excursions after an oral gavage glucose tolerance test $(2 \mathrm{~g} / \mathrm{kg}$ LBM) with corresponding incremental area under the curve (iAUC) (E, F and G) at 5 weeks post AAV treatment $(n=3-$ 12) and (H, I and J) 10 weeks post AAV-delivery (n=6-15). Fasting blood glucose was taken after a 6 hour fast and is the ' 0 ' time value represented in the OGTT. Diet effects between NC and HFD $* \mathrm{p}=<0.05 * * * \mathrm{p}=<0.001, \# \mathrm{p}=<0.05$ treatment effect. Two-way ANOVA used for comparisons. Graphs indicate mean \pm SEM.

Fig 5. Aspects of whole-body energy metabolism measured in a CLAMs system for AAV:CON and AAV:Hsp72 treated mice fed either a NC or HFD. (A) Raw oxygen consumption (VO ør langalictstedluring light cycle, dark cycle and over the total 24 hour measurement period (B), VO 2(C)dribal ised tQ ledatめody $n$ normalised to body weight. (D) Correlation between unadjusted $\mathrm{VO}_{2}$ (total $24 \mathrm{hr}$ period) to body weight, (E) lean mass and (F) fat mass. (G) Respiratory exchange ratio (RER). (H) 
Energy expenditure (Heat). (I) Total movement/activity as measured via beam breaks $(\mathrm{x}+\mathrm{Y}$ ambulatory plus z breaks). Diet effect between NC and HFD. ${ }^{* * *} \mathrm{p}=<0.001, \mathrm{n}=3-12$. . Twoway ANOVA used for comparisons. Graphs indicate mean $\pm \mathrm{SEM}$.

Supp Fig 1. Images and quantification of insulin signalling cascade in skeletal muscles obtained from the intramuscular AAV experiments. (A) Blots demonstrating bands corresponding to pAKT Ser473, total-AKT, pGSK-3 $\pm^{2}$ Ser 21/9, total GSK-3, and GAPDH. (B) Quantification of densitometry for AKT levels, (D) Quantification of densitometry for GSK levels. $n=3-5$ per group. Graphs indicate mean \pm SEM.

Supp Fig 2. Comparison of skeletal muscle Hsp72 expression levels with different models. (A) Skeletal muscle Hsp72 levels from Hsp72Tg, AAV:Hsp72 (IM) and AAV:Hsp72 (systemic) experiments compared to wildtype or AAV:CON treated skeletal muscle. (B) Direct side by side comparison of Hsp72 levels from Hsp72Tg, AAV:Hsp72 (IM) and AAV:Hsp72 (systemic) conditions.

Supp Fig 3. Flow diagram of animal use and analysis for the Intramuscular (IM) intravenous insulin tolerance test (ivITT) experiments based on the Consolidated Standards or Animal Experiment ReporTing (CONSAERT) template.

This article is protected by copyright. All rights reserved. 
Supp Fig 4. Flow diagram of animal use and analysis for the metabolic systemic AAV intervention experiments based on the Consolidated Standards or Animal Experiment ReporTing (CONSAERT) template.

\section{References}

This article is protected by copyright. All rights reserved. 
1. Shaw JE, Sicree RA, Zimmet PZ. Global estimates of the prevalence of diabetes for 2010 and 2030. Diabetes Res Clin Pract. 2010;87(1):4-14.

2. Whiting DR, Guariguata L, Weil C, Shaw J. IDF diabetes atlas: global estimates of the prevalence of diabetes for 2011 and 2030. Diabetes Res Clin Pract. 2011;94(3):311-321.

3. Kurucz I, Morva A, Vaag A, et al. Decreased expression of heat shock protein 72 in skeletal muscle of patients with type 2 diabetes correlates with insulin resistance. Diabetes. 2002;51(4):1102-1109.

4. Bruce CR, Carey AL, Hawley JA, Febbraio MA. Intramuscular heat shock protein 72 and heme oxygenase- 1 mRNA are reduced in patients with type 2 diabetes: evidence that insulin resistance is associated with a disturbed antioxidant defense mechanism. Diabetes. 2003;52(9):2338-2345.

5. Chung J, Nguyen AK, Henstridge DC, et al. HSP72 protects against obesity-induced insulin resistance. Proc Natl Acad Sci U S A. 2008;105(5):1739-1744.

6. Rodrigues-Krause J, Krause M, O'Hagan C, et al. Divergence of intracellular and extracellular HSP72 in type 2 diabetes: does fat matter? Cell Stress Chaperones. 2012;17(3):293-302.

7. Morino S, Kondo T, Sasaki K, et al. Mild electrical stimulation with heat shock ameliorates insulin resistance via enhanced insulin signaling. PLoS One. 2008;3(12):e4068.

8. Kondo T, Ono K, Kitano S, et al. Mild Electrical Stimulation with Heat Shock Reduces Visceral Adiposity and Improves Metabolic Abnormalities in Subjects with Metabolic Syndrome or Type 2 Diabetes: Randomized Crossover Trials. EBioMedicine. 2014;1(1):80-89.

9. Rogers RS, Morris EM, Wheatley JL, et al. Deficiency in the Heat Stress Response Could Underlie Susceptibility to Metabolic Disease. Diabetes. 2016;65(11):33413351.

10. Gupte AA, Bomhoff GL, Swerdlow RH, Geiger PC. Heat treatment improves glucose tolerance and prevents skeletal muscle insulin resistance in rats fed a high-fat diet. Diabetes. 2009;58(3):567-578.

11. Gupte AA, Bomhoff GL, Touchberry CD, Geiger PC. Acute heat treatment improves insulin-stimulated glucose uptake in aged skeletal muscle. J Appl Physiol (1985). 2011;110(2):451-457.

12. Karpe PA, Tikoo K. Heat shock prevents insulin resistance-induced vascular complications by augmenting angiotensin-(1-7) signaling. Diabetes. 2014;63(3):11241139.

13. Zeng $\mathrm{XY}$, Wang $\mathrm{H}$, Bai $\mathrm{F}$, et al. Identification of matrine as a promising novel drug for hepatic steatosis and glucose intolerance with HSP72 as an upstream target. $\mathrm{Br} J$ Pharmacol. 2015;172(17):4303-4318.

14. Adachi H, Kondo $\mathrm{T}$, Ogawa $\mathrm{R}$, et al. An acylic polyisoprenoid derivative, geranylgeranylacetone protects against visceral adiposity and insulin resistance in high-fat-fed mice. Am J Physiol Endocrinol Metab. 2010;299(5):E764-771. 
15. Kavanagh K, Flynn DM, Jenkins KA, Zhang L, Wagner JD. Restoring HSP70 deficiencies improves glucose tolerance in diabetic monkeys. Am $J$ Physiol Endocrinol Metab. 2011;300(5):E894-901.

16. Literati-Nagy Z, Tory K, Literati-Nagy B, et al. The HSP co-inducer BGP-15 can prevent the metabolic side effects of the atypical antipsychotics. Cell Stress Chaperones. 2012;17(4):517-521.

17. Henstridge DC, Bruce CR, Drew BG, et al. Activating HSP72 in rodent skeletal muscle increases mitochondrial number and oxidative capacity and decreases insulin resistance. Diabetes. 2014;63(6):1881-1894.

18. Drew BG, Ribas V, Le JA, et al. HSP72 is a mitochondrial stress sensor critical for Parkin action, oxidative metabolism, and insulin sensitivity in skeletal muscle. Diabetes. 2014;63(5):1488-1505.

19. Chen HW, Chen SC, Tsai JL, Yang RC. Previous hyperthermic treatment increases mitochondria oxidative enzyme activity and exercise capacity in rats. Kaohsiung $J$ Med Sci. 1999;15(10):572-580.

20. Chen HW, Hsu C, Lu TS, Wang SJ, Yang RC. Heat shock pretreatment prevents cardiac mitochondrial dysfunction during sepsis. Shock. 2003;20(3):274-279.

21. Liu CT, Brooks GA. Mild heat stress induces mitochondrial biogenesis in $\mathrm{C} 2 \mathrm{C} 12$ myotubes. J Appl Physiol (1985). 2012;112(3):354-361.

22. Drucker DJ. Never Waste a Good Crisis: Confronting Reproducibility in Translational Research. Cell Metab. 2016;24(3):348-360.

23. Marber MS, Mestril R, Chi SH, Sayen MR, Yellon DM, Dillmann WH. Overexpression of the rat inducible $70-\mathrm{kD}$ heat stress protein in a transgenic mouse increases the resistance of the heart to ischemic injury. $J$ Clin Invest. 1995;95(4):1446-1456.

24. Salva MZ, Himeda CL, Tai PW, et al. Design of tissue-specific regulatory cassettes for high-level rAAV-mediated expression in skeletal and cardiac muscle. Mol Ther. 2007;15(2):320-329.

25. Gregorevic P, Blankinship MJ, Allen JM, et al. Systemic delivery of genes to striated muscles using adeno-associated viral vectors. Nat Med. 2004;10(8):828-834.

26. Winbanks CE, Beyer C, Qian H, Gregorevic P. Transduction of skeletal muscles with common reporter genes can promote muscle fiber degeneration and inflammation. PLoS One. 2012;7(12):e51627.

27. Henstridge DC, Estevez E, Allen TL, et al. Genetic manipulation of cardiac Hsp72 levels does not alter substrate metabolism but reveals insights into high-fat feedinginduced cardiac insulin resistance. Cell Stress Chaperones. 2015.

28. Henstridge DC, Bruce CR, Pang CP, et al. Skeletal muscle-specific overproduction of constitutively activated c-Jun N-terminal kinase (JNK) induces insulin resistance in mice. Diabetologia. 2012;55(10):2769-2778.

29. Fueger PT, Bracy DP, Malabanan CM, Pencek RR, Wasserman DH. Distributed control of glucose uptake by working muscles of conscious mice: roles of transport and phosphorylation. Am J Physiol Endocrinol Metab. 2004;286(1):E77-84.

30. Hooper PL. Hot-tub therapy for type 2 diabetes mellitus. $N$ Engl $J$ Med. 1999;341(12):924-925. 
31. Literati-Nagy B, Kulcsar E, Literati-Nagy Z, et al. Improvement of insulin sensitivity by a novel drug, BGP-15, in insulin-resistant patients: a proof of concept randomized double-blind clinical trial. Horm Metab Res. 2009;41(5):374-380.

32. Kowalski GM, Bruce CR. The regulation of glucose metabolism: implications and considerations for the assessment of glucose homeostasis in rodents. Am J Physiol Endocrinol Metab. 2014;307(10):E859-E871.

This article is protected by copyright. All rights reserved. 

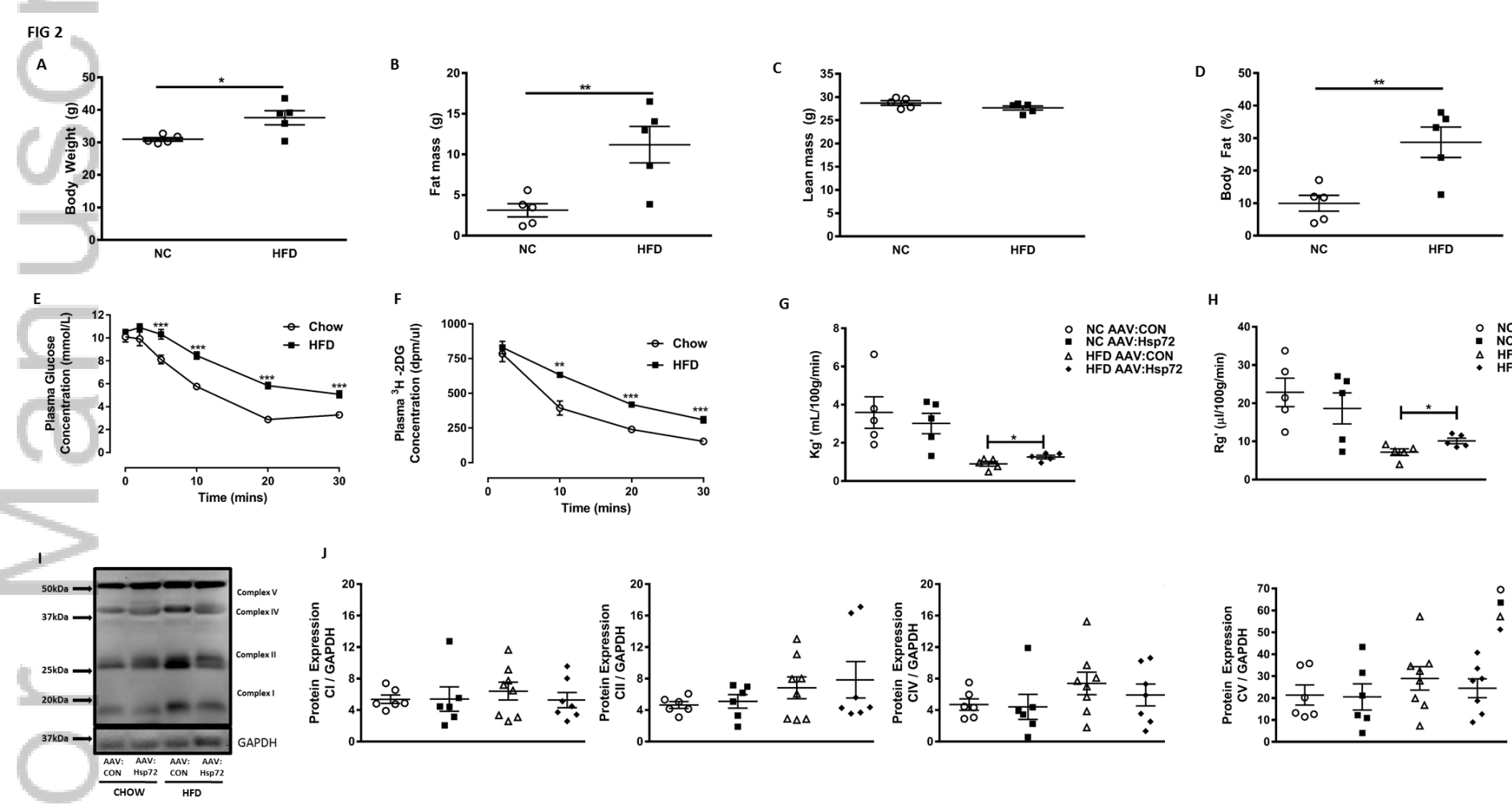

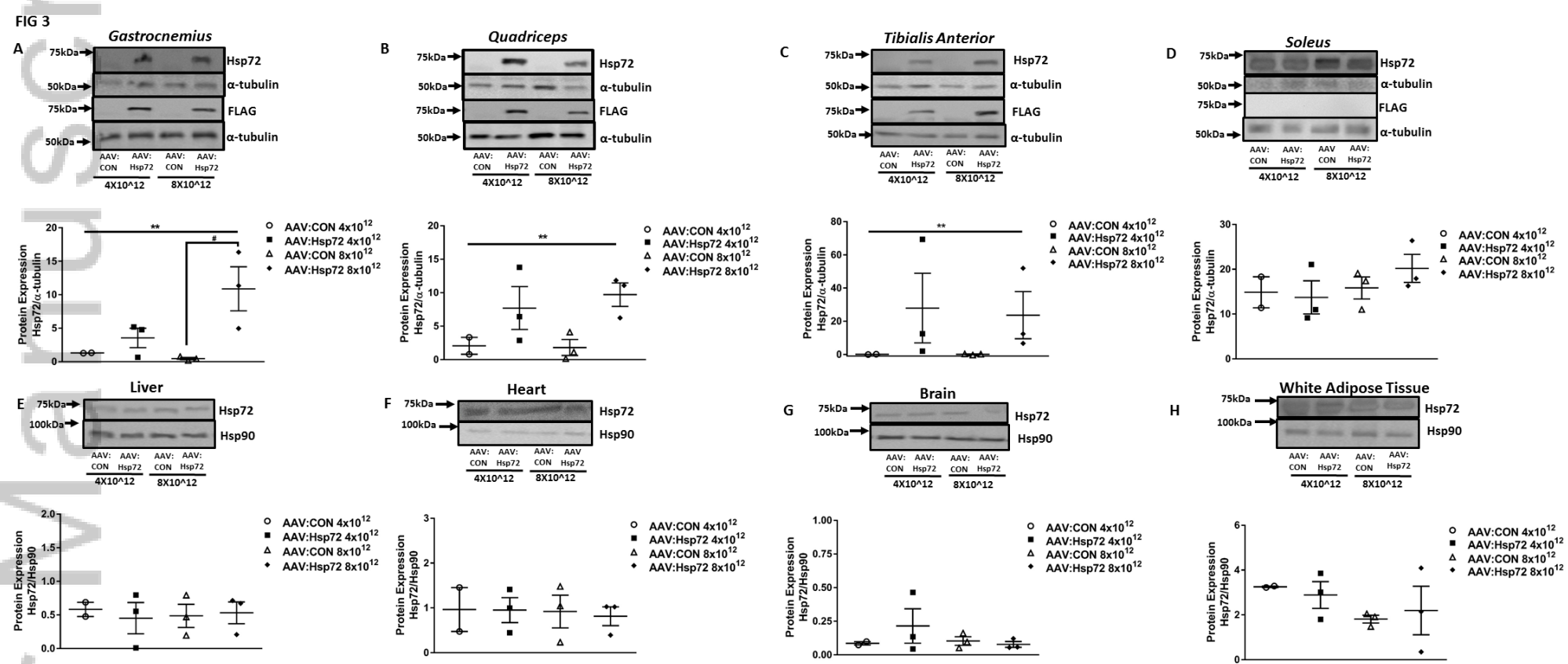

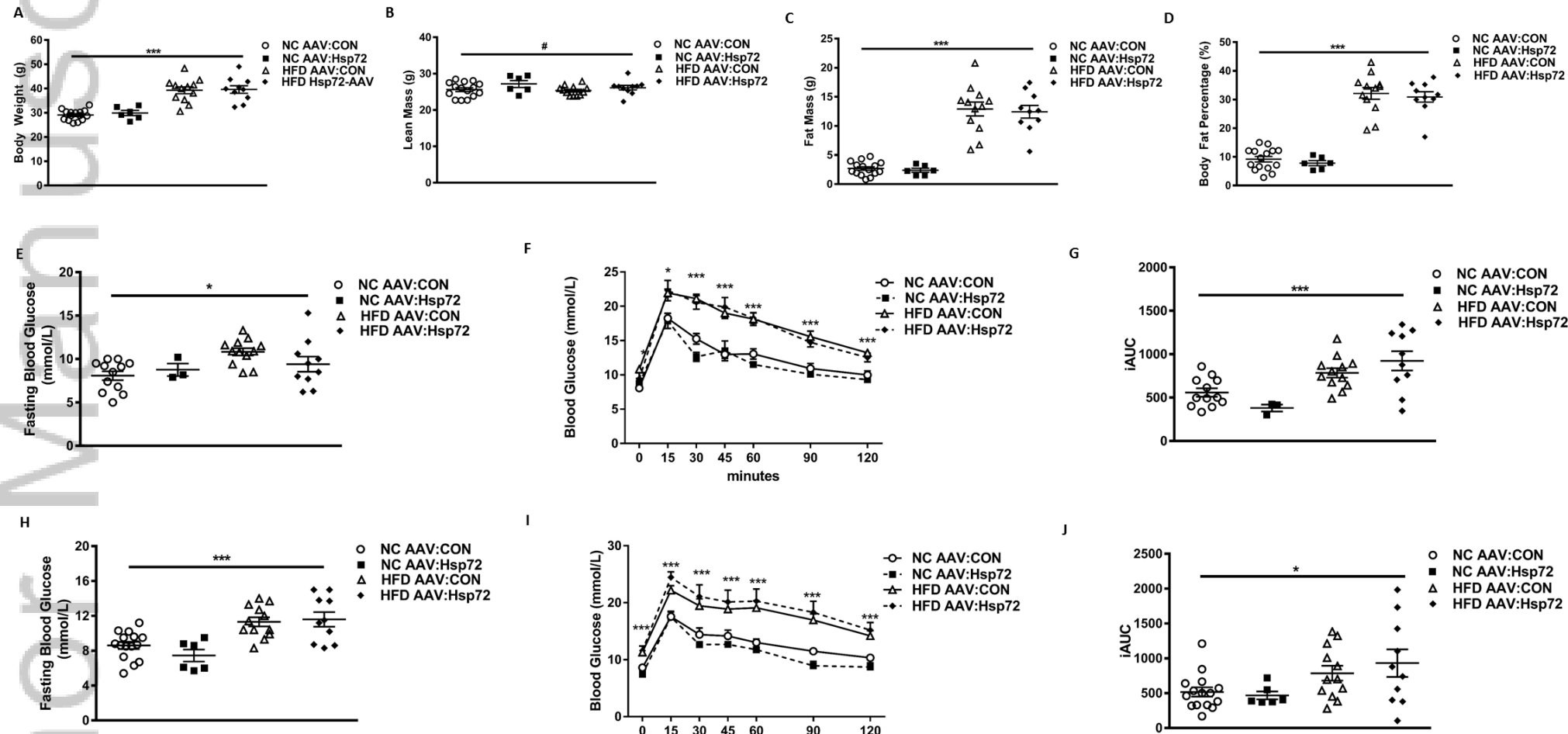

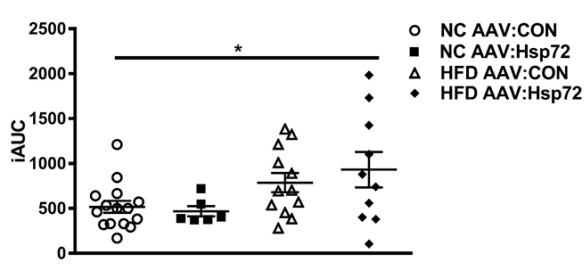


FIG 5
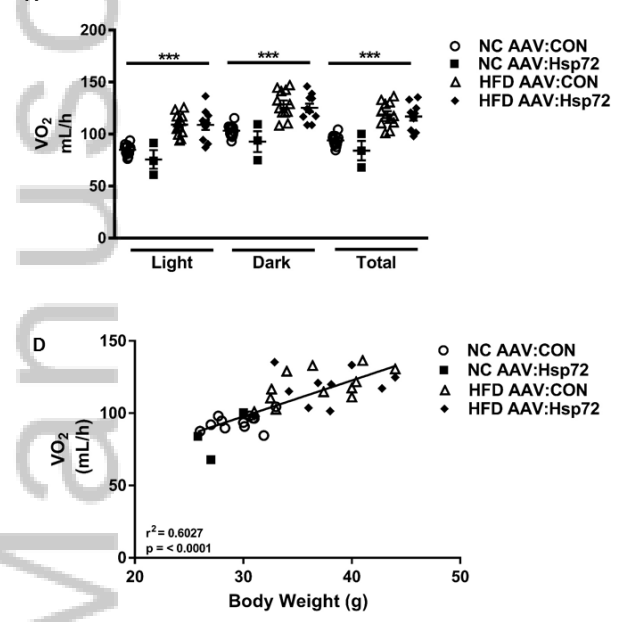

G

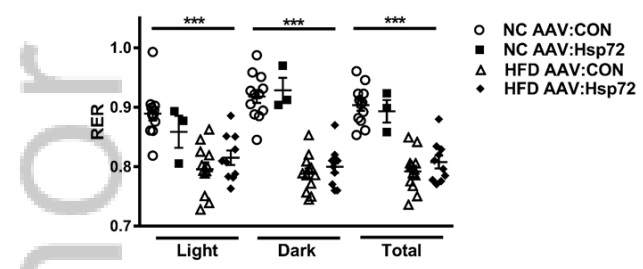

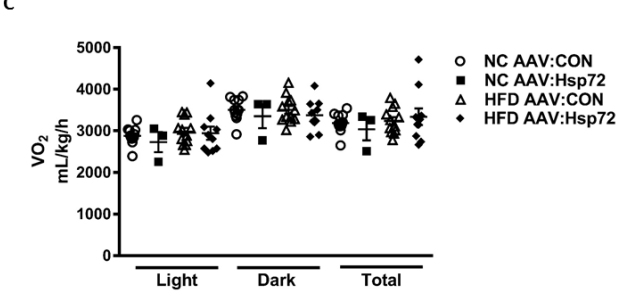

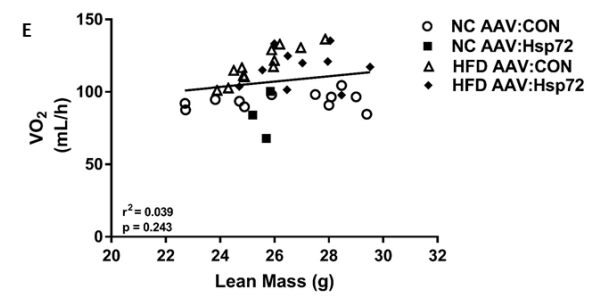

H

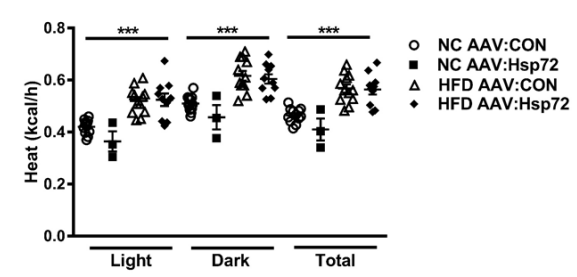

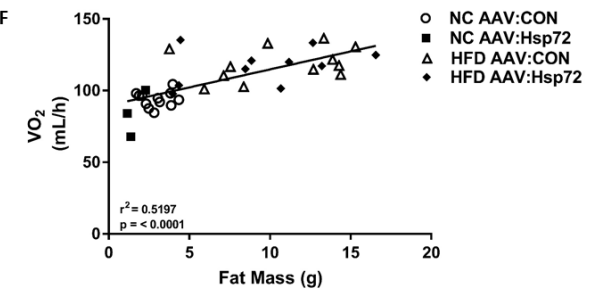

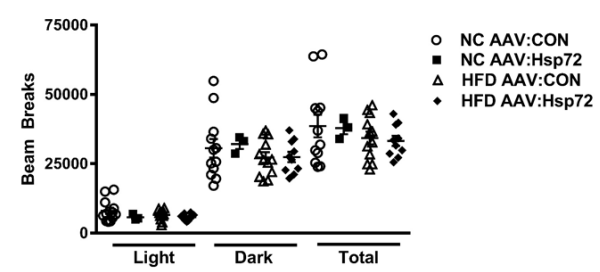




\section{University Library}

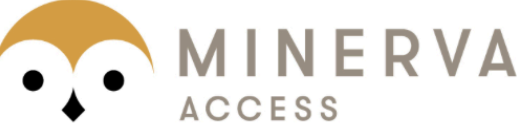

A gateway to Melbourne's research publications

Minerva Access is the Institutional Repository of The University of Melbourne

Author/s:

Marshall, JPS;Estevez, E;Kammoun, HL;King, EJ;Bruce, CR;Drew, BG;Qian, H;lliades, P;Gregorevic, P;Febbraio, MA;Henstridge, DC

Title:

Skeletal muscle-specific overexpression of heat shock protein 72 improves skeletal muscle insulin-stimulated glucose uptake but does not alter whole body metabolism

Date:

2018-08-01

Citation:

Marshall, J. P. S., Estevez, E., Kammoun, H. L., King, E. J., Bruce, C. R., Drew, B. G., Qian, H., Iliades, P., Gregorevic, P., Febbraio, M. A. \& Henstridge, D. C. (2018). Skeletal muscle-specific overexpression of heat shock protein 72 improves skeletal muscle insulinstimulated glucose uptake but does not alter whole body metabolism. DIABETES OBESITY \& METABOLISM, 20 (8), pp.1928-1936. https://doi.org/10.1111/dom.13319.

Persistent Link:

http://hdl.handle.net/11343/283941 